\title{
Clinical significance of peripheral blood- derived inflammation markers in advanced gastric cancer after radical resection
}

\author{
Lihu Gu${ }^{1,2,3+}$, Mian Wang ${ }^{4 \dagger}$, Xuena Cui ${ }^{5}$, Jiahang $\mathrm{Mo}^{6}$, Lingling Yuan ${ }^{1}$, Feiyan Mao ${ }^{1}$, Kang Zhang ${ }^{7}$,
}

Derry Minyao $\mathrm{Ng}^{7}$, Ping Chen ${ }^{1}$ and Dongjie Wang ${ }^{*^{*}}$ (I)

\begin{abstract}
Background: The prognostic significance of peripheral blood-derived inflammation markers in patients with gastric cancer (GC) has not been elucidated. This study aimed to investigate the relationship between systemic inflammatory markers and GC prognosis.

Methods: A prospective observational cohort study involving 598 patients was conducted to analyze the prognosis of GC based on systemic inflammatory markers. The following peripheral blood-derived inflammation markers were evaluated: the neutrophil-lymphocyte ratio (NLR), platelet lymphocyte ratio (PLR), systemic immune-inflammation index (SII), C-reactive protein/albumin (CRP/Alb) ratio, Glasgow Prognostic Score (GPS), modified Glasgow Prognostic Score (mGPS), prognostic nutrition index (PNI), and prognostic index (PI). The receiver operating characteristics $(\mathrm{ROC})$ curve and the Youden index were used to determine the optimal cutoff values. Univariate and multivariate analysis of prognostic factors was conducted accordingly.

Results: The optimal cutoff values of the PNI, fibrinogen, NLR, PLR, SIl, and CRP/Alb were 49.5, 397 ng/dl, 2.5, 154, 556, and 0.05 , respectively. Multivariate analysis showed that age, PLR, TNM stage, and chemotherapy were the independent prognostic factors for advanced gastric cancer (AGC). Adjuvant chemotherapy improved the long-term prognosis of patients with PLR $\geq 154$, but chemotherapy had no significant effect on the survival of patients with PLR $<154$.

Conclusions: Our findings show that higher PLR $(\geq 154)$ is an independent risk factor for poor prognosis in GC patients. Besides, PLR can predict adjuvant chemotherapy (oxaliplatin/5-fluorouracil combination) response in patients with GC after surgery.
\end{abstract}

Keywords: Gastric cancer, Systemic inflammatory marker, Platelet lymphocyte ratio, Prognosis, Chemotherapy

\section{Background}

Gastric cancer (GC) is one of the most common malignant tumors and that poses a serious threat to human health, especially in Asia. Approximately 300,000 deaths and 400,000 new cases of GC occur in China every year

\footnotetext{
* Correspondence: 0574wdj@sina.com

${ }^{+}$Lihu Gu and Mian Wang contributed equally to this work.

${ }^{8}$ Department of Clinical Laboratory, HwaMei Hospital, University of Chinese Academy of Sciences, Northwest Street 41, Haishu District, Ningbo 315010, Zhejiang, China

Full list of author information is available at the end of the article
}

[1]. Despite the advancement in diagnostic and treatment methods, the prognosis of advanced gastric cancer (AGC) patients has remained poor [2]. Tumor, Node, Metastasis (TNM) staging based on the International Union Against Cancer (UICC)/American Joint Committee on Cancer (AJCC) guidelines is currently the standard approach of determining the prognosis of GC patients [3]. However, several prognostic factors related to $\mathrm{GC}$ have been proposed, which include peripheral blood-derived inflammation markers, such as neutrophil-lymphocyte ratio (NLR),

(c) The Author(s). 2020 Open Access This article is licensed under a Creative Commons Attribution 4.0 International License, which permits use, sharing, adaptation, distribution and reproduction in any medium or format, as long as you give appropriate credit to the original author(s) and the source, provide a link to the Creative Commons licence, and indicate if changes were made. The images or other third party material in this article are included in the article's Creative Commons licence, unless indicated otherwise in a credit line to the material. If material is not included in the article's Creative Commons licence and your intended use is not permitted by statutory regulation or exceeds the permitted use, you will need to obtain permission directly from the copyright holder. To view a copy of this licence, visit http://creativecommons.org/licenses/by/4.0/. The Creative Commons Public Domain Dedication waiver (http://creativecommons.org/publicdomain/zero/1.0/) applies to the data made available in this article, unless otherwise stated in a credit line to the data. 
platelet lymphocyte ratio (PLR), systemic immuneinflammation index (SII), C-reactive protein/albumin (CRP/ $\mathrm{Alb})$ ratio, and Glasgow Prognostic Score (GPS) [4-6].

Some studies have combined the TNM staging system with GC related risk factors to improve the accuracy of the long-term prognosis of the disease [7]. Routine peripheral blood-derived inflammation markers are closely associated with the pathogenesis of GC [8]. Moreover, the use of these markers as prognostic factors is advantageous because most of the peripheral blood-derived inflammation markers belong to the routine test items; the test cost is cheap and does not require special equipment.

Park indicated that preoperative body mass index (BMI) and prognostic nutritional index (PNI), as well as their postoperative changes, are related to the prognosis of stage II/III GC [9]. Also, Jagadesham reported that the combination of one or more markers of systemic inflammation could precisely predict the prognosis of advanced adenocarcinoma of the esophagus [10]. Studies have suggested that combining NLR and PLR could significantly improve the accuracy of predicting the firstline chemosensitivity in AGC [11]. Contrarily, Xu et al. indicated that CRP/Alb might be a promising predictor of early recurrence (recurrence within 12 months after radical gastrectomy) and postoperative chemotherapy in stage III GC [12].

Unfortunately, most of these findings are based on small sample retrospective studies with insufficient evidence, which could be the reason for the inconsistencies among the various reports. Herein, we designed a prospective observational cohort to examine the relationship between peripheral blood-derived inflammation markers and the prognosis of GC. Also, we hypothesized that derangements in one or more systemic inflammation markers may be associated with poor disease outcomes and the ineffectiveness of chemotherapy.

\section{Methods}

\section{Study population}

This was a prospective observational cohort study involving patients who underwent radical gastrectomy from January 2013 to December 2016 at HwaMei Hospital, University of Chinese Academy of Sciences, and was approved by the Ethics Committee of the HwaMei Hospital and the University of Chinese Academy of Sciences (approval NO. PJ-NBEY-KY-2019-153-01). Written consent was obtained from all patients before enrollment. The inclusion criteria are as follows: (1) patients with histologically proven primary adenocarcinoma of the stomach; (2) patients without a history of gastrectomy or any other malignant tumor; (3) patients negative for any inflammatory or hematological diseases; (4) patients with pathologically negative resection margins (R0, 5) patients not receiving any neoadjuvant chemoradiotherapy; (6) follow-up period of at least 36 months. A treatment plan that includes a 5-fluorouracil (5-FU)-based or any platinum-based adjuvant chemotherapy is recommended for all patients diagnosed with stage II-III of the disease [13].

\section{Systemic inflammatory markers and histological examination}

The whole blood and clinicopathological data were obtained 1 week before initial treatment. Blood samples were collected for routine laboratory tests, which included complete blood count, CRP, albumin, fibrinogen, and tumor markers, such as carcinoembryonic antigen (CEA). The following common peripheral blood-derived inflammation markers were included based on previous studies: NLR, PLR, SII, CRP/Alb, GPS, modified Glasgow Prognostic Score (mGPS), PNI, and prognostic index (PI).

All surgical resection specimens were assessed according to the handling guideline of the third edition of the Japanese classification of gastric carcinoma. And the staging was conducted by pathologists using the 8th edition of the UICC/AJCC TNM staging system [3].

\section{Follow-up}

Patients included in the study were followed up with every 3-6 months for the first 2 years and then annually after until death or at least 5 years after the surgery. Disease-free survival (DFS) was defined as the time from surgery to death or until the patient is found to have either loco-regional recurrence or distant recurrence. Disease-specific survival (DSS) was defined as the time from surgery to death as a result of GC. Patients with no records of the above events were censored at the date of their last known contact. The median follow-up period for the entire cohort was 50 months (range 4-83 months), and all patient follow-up was stopped in December 2019.

\section{Statistical analysis}

The continuous variables in the study were analyzed using either the independent sample $t$-test or Wilcoxon rank-sum test, while the categorical variables were analyzed using either the Pearson's chi-squared test or Fisher's exact test where applicable. The receiver operating characteristics (ROC) curve was calculated, and the Youden index (maximum $=$ sensitivity + specificity -1$)$ was used to determine the optimal cutoff value for the number of lymph nodes retrieved, PNI, fibrinogen, NLR, PLR, SII, and CRP/Alb. Any potentially relevant factors derived from the univariate analysis were assessed in the multivariate model using Cox's regression. We also calculated the hazard ratios (HR) and 95\% confidence intervals (CI). The DFS and DSS rate were obtained using the Kaplan-Meier method, and the log-rank test was used to 
determine if the result was statistically significant. A $p<$ 0.05 was considered statistically significant, and all statistical tests were performed 2-sided. SPSS software (version 25.0, SPSS Inc. IL, USA) was used for all analyses.

\section{Results}

A total of 598 patients were recruited from January 2013 to December 2016. The 5-year DFS and DSS rates of all patients were 72.6 and $75.4 \%$, respectively. Male patients were about twice as many as female patients, and the tumors in the distal stomach accounted for $77 \%$ of all the tumors. Concerning the GC staging, patients with GC stage I, II, and III were 119,113 , and 366, respectively. The 5-year DFS and DSS rates after surgery for stage I patients was 97 and 98\%, respectively, whereas, for stage II patients, the rates were 81.4 and $85.8 \%$, respectively. For stage III patients, the rates were 52.1 and $55.6 \%$, respectively (Supplementary Table 1).

A total of 376 patients received adjuvant chemotherapy, of which 239 received SOX regimen [14] and 112 XELOX [15]. The remaining 25 patients received other chemotherapy treatments [16].

\section{Optimal cutoff analysis}

The optimal cutoff value of the number of lymph nodes retrieved, PNI, fibrinogen, NLR, PLR, SII, and CRP/Alb were $30,49.5,397 \mathrm{ng} / \mathrm{dl}, 2.5,154,556$, and 0.05 , respectively (Supplementary Table 2).

\section{Clinicopathological factors and survival analysis}

A total of 23 potential risk factors were selected, and from the univariate analysis results, we found 17 clinicopathological characteristics that were significantly associated with the 5-year DFS rate in all enrolled patients, the clinicopathological factors are as follows: age, tumor location, type of gastrectomy, tumor size, perineural invasion, lymphovascular invasion, $\mathrm{T}$ stage, $\mathrm{N}$ stage, chemotherapy, PNI, fibrinogen, NLR, PLR, SII, GPS, CRP/Alb, and CEA (Table 1). The multivariate Cox proportional hazards model analysis determined that age, $\mathrm{T}$ stage, $\mathrm{N}$ stage, number of lymph nodes retrieved, and PLR were independent prognostic factors for GC (Table 1).

\section{Risk factors for advanced gastric cancer}

As there is a close correlation between the TNM stage and the 5-year DFS, we hypothesis that the number of lymph nodes extracted may be associated with the prognosis. If the total number of Lymph nodes extracted is less than 16, it would be defined as inadequate lymph node dissection, which is especially important for AGC. Therefore, only patients with the AGC lymph nodes $\geq 16$ were included in the analysis. Multivariate analysis showed that age, PLR, and TNM stages were associated with 5-year DFS. Also, age, PLR, TNM stage, and chemotherapy were associated with 5-year DSS (Table 2).

Risk factors associated with stage II and III gastric cancer The Kaplan-Meier curves were used to determine the long-term disease outcomes of GC patients in stage II and stage III. In GC stage II, the 5-year DFS rates were similar among patients with age $\leq 60$ and age $>60(p=$ $0.213)$. However, in stage III, the 5 -year DFS rate of GC patients aged $>60$ was worse than those aged $\leq 60$ ( $p=$ 0.016). Similarly, in GC stage III, the 5-year DFS rate of patients with PLR $\geq 154$ was worse than those with PLR $<154(p<0.001)$. But this phenomenon was not observed in GC stage II $(p=0.153)$. The effect of chemotherapy on the prognosis of patients with stage II GC was not statistically significant $(p=0.260)$. In contrast, chemotherapy significantly improved the 5-year DFS in patients with stage III GC ( $p=0.017)$ (Fig. 1).

With the 5-year DSS as a long-term prognostic index, age, PLR, and chemotherapy had the same effect on the prognosis of stage II and III GC patients as 5-year DFS, except that chemotherapy also improved the 5-year DSS of stage II GC patients ( $p=0.033)$ (Fig. 2).

When considered together with the above observations, PLR was found to be an independent risk factor for the prognosis of AGC. Patients with AGC were divided into either the PLR $<154$ and PLR $\geq 154$ subgroups. Further analysis of the clinicopathological factors between the two groups revealed that tumor lesions in the PLR < 154 group were smaller than those in the PLR $\geq 154$ group. But there was no other difference between the groups (Table 3). Further analysis of the relationship between chemotherapy and PLR showed that chemotherapy improved the long-term prognosis of patients in the PLR $\geq 154$ group, including 5-year DFS and DSS ( $p=0.026, p=0.014$, respectively). Notably, chemotherapy had no significant effect on the long-term prognosis of patients in the PLR < 154 group (Fig. 3).

\section{Discussion}

Many studies have been conducted to investigate the correlation between peripheral blood-derived inflammation markers and tumor prognosis [17, 18]. Liu showed that CRP/Alb was an independent prognostic marker for patients with ovarian cancer [19]. Also, the NLR and PLR are prognostic factors in patients with non-small cell lung cancer after stereotactic radiation therapy [20]. The independent risk factors for poor GC prognosis include NLR, PLR, fibrinogen, PNI, GPS, CRP/Alb, among others. Also, some studies have combined these systemic inflammatory markers with or without TNM stage to provide new prognostic tools [21-23]. However, most of these studies were retrospective, and reported 
Table 1 Univariate and multivariate analysis of prognostic factors in patients with gastric cancer

\begin{tabular}{|c|c|c|c|c|c|c|}
\hline \multirow{2}{*}{$\begin{array}{l}\text { Clinicopathological } \\
\text { feature }\end{array}$} & \multicolumn{3}{|c|}{ Univariate analysis } & \multicolumn{3}{|c|}{ Multivariate analysis } \\
\hline & $\mathrm{HR}$ & $95 \% \mathrm{Cl}$ & $p$ value & $\mathrm{HR}$ & $95 \% \mathrm{Cl}$ & $p$ value \\
\hline \multicolumn{7}{|l|}{ Age (years) } \\
\hline$\leq 60$ & 1 & & & 1 & & \\
\hline$>60$ & 1.62 & $1.16-2.25$ & 0.005 & 1.63 & $1.15-2.31$ & 0.007 \\
\hline \multicolumn{7}{|l|}{ Gender } \\
\hline Male & 1 & & & & & \\
\hline Female & 1.01 & $0.73-1.40$ & 0.967 & & & \\
\hline \multicolumn{7}{|l|}{ BMI } \\
\hline$\leq 24 \mathrm{~kg} / \mathrm{m}^{2}$ & 1 & & & & & \\
\hline$>24 \mathrm{~kg} / \mathrm{m}^{2}$ & 0.77 & $0.52-1.13$ & 0.175 & & & \\
\hline \multicolumn{7}{|l|}{ Tumor location } \\
\hline Upper third & 1 & & & & & \\
\hline Middle third & 0.53 & $0.29-0.98$ & 0.041 & & & \\
\hline Lower third & 0.47 & $0.31-0.71$ & $<0.001$ & & & \\
\hline Two-thirds or more & 0.76 & $0.27-2.15$ & 0.600 & & & \\
\hline \multicolumn{7}{|l|}{ Gastrectomy } \\
\hline Distal & 1 & & & & & \\
\hline Total & 1.57 & $1.10-2.23$ & 0.013 & & & \\
\hline Proximal & NA & & & & & \\
\hline \multicolumn{7}{|l|}{ Tumor size } \\
\hline$\leq 5 \mathrm{~cm}$ & 1 & & & & & \\
\hline$>5 \mathrm{~cm}$ & 2.60 & $1.90-3.54$ & $<0.001$ & & & \\
\hline \multicolumn{7}{|l|}{ Histologic type } \\
\hline Differentiated & 1 & & & & & \\
\hline Undifferentiated & 1.29 & $0.95-1.76$ & 0.106 & & & \\
\hline \multicolumn{7}{|l|}{ Perineural invasion } \\
\hline Absence & 1 & & & & & \\
\hline Presence & 2.73 & $2.00-3.72$ & $<0.001$ & & & \\
\hline \multicolumn{7}{|l|}{ Lymphovascular invasion } \\
\hline Absence & 1 & & & & & \\
\hline Presence & 2.78 & $2.02-3.83$ & $<0.001$ & & & \\
\hline \multicolumn{7}{|l|}{ T category } \\
\hline $\mathrm{T} 1$ & 1 & & & 1 & & \\
\hline $\mathrm{T} 2$ & 4.30 & $1.56-11.83$ & 0.005 & 3.04 & $1.08-8.57$ & 0.036 \\
\hline T3 & 2.04 & $0.25-16.92$ & 0.510 & 1.69 & $0.20-14.50$ & 0.633 \\
\hline T4a & 15.79 & $6.97-35.76$ & $<0.001$ & 8.22 & $3.26-20.75$ & $<0.001$ \\
\hline $\mathrm{T} 4 \mathrm{~b}$ & 28.21 & $9.78-81.36$ & $<0.001$ & 13.05 & $3.98-42.82$ & $<0.001$ \\
\hline \multicolumn{7}{|l|}{$\mathrm{N}$ category } \\
\hline No & 1 & & & 1 & & \\
\hline N1 & 3.22 & $1.76-5.90$ & $<0.001$ & 1.70 & $0.88-3.26$ & 0.112 \\
\hline N2 & 4.71 & $2.75-8.05$ & $<0.001$ & 2.09 & $1.15-3.81$ & 0.016 \\
\hline N3a & 11.35 & $6.82-18.89$ & $<0.001$ & 5.38 & $3.00-9.65$ & $<0.001$ \\
\hline N3b & 24.19 & $13.27-44.07$ & $<0.001$ & 11.01 & $5.50-22.04$ & $<0.001$ \\
\hline
\end{tabular}


Table 1 Univariate and multivariate analysis of prognostic factors in patients with gastric cancer (Continued)

\begin{tabular}{|c|c|c|c|c|c|c|}
\hline \multirow{2}{*}{$\begin{array}{l}\text { Clinicopathological } \\
\text { feature }\end{array}$} & \multicolumn{3}{|c|}{ Univariate analysis } & \multicolumn{3}{|c|}{ Multivariate analysis } \\
\hline & $\mathrm{HR}$ & $95 \% \mathrm{Cl}$ & $p$ value & HR & $95 \% \mathrm{Cl}$ & $p$ value \\
\hline No & 1 & & & 1 & & \\
\hline Yes & 1.58 & $1.12-2.22$ & 0.009 & 0.70 & $0.48-1.01$ & 0.059 \\
\hline \multicolumn{7}{|c|}{ Number of lymph nodes retrieved } \\
\hline$\leq 15$ & 1 & & & 1 & & \\
\hline $16-29$ & 0.75 & $0.50-1.13$ & 0.169 & 0.46 & $0.30-0.70$ & $<0.001$ \\
\hline$\geq 30$ & 1.36 & $0.88-2.11$ & 0.166 & 0.52 & $0.32-0.84$ & 0.008 \\
\hline \multicolumn{7}{|l|}{ PI } \\
\hline 0 & 1 & & & & & \\
\hline 1 & 1.54 & $0.96-2.49$ & 0.075 & & & \\
\hline \multicolumn{7}{|l|}{ PNI } \\
\hline$<49.5$ & 1 & & & & & \\
\hline$\geq 49.5$ & 0.40 & $0.27-0.59$ & $<0.001$ & & & \\
\hline \multicolumn{7}{|l|}{ Fibrinogen } \\
\hline$<397$ ng/dl & 1 & & & & & \\
\hline$\geq 397 \mathrm{ng} / \mathrm{dl}$ & 2.06 & $1.51-2.81$ & $<0.001$ & & & \\
\hline \multicolumn{7}{|l|}{ NLR } \\
\hline$<2.5$ & 1 & & & 1 & & \\
\hline$\geq 2.5$ & 1.69 & $1.24-2.31$ & 0.001 & 0.70 & $0.48-1.01$ & 0.059 \\
\hline \multicolumn{7}{|l|}{ PLR } \\
\hline$<154$ & 1 & & & 1 & & \\
\hline$\geq 154$ & 2.21 & $1.62-3.01$ & $<0.001$ & 1.70 & $1.20-2.42$ & 0.003 \\
\hline \multicolumn{7}{|l|}{ SII } \\
\hline$<556$ & 1 & & & & & \\
\hline$\geq 556$ & 1.94 & $1.43-2.64$ & $<0.001$ & & & \\
\hline \multicolumn{7}{|l|}{ GPS } \\
\hline 0 & 1 & & & & & \\
\hline 1 & 1.76 & $1.24-2.49$ & 0.002 & & & \\
\hline 2 & 1.89 & $1.04-3.43$ & 0.037 & & & \\
\hline \multicolumn{7}{|l|}{ mGPS } \\
\hline 0 & 1 & & & & & \\
\hline 1 & 1.53 & $0.68-3.46$ & 0.307 & & & \\
\hline 2 & 1.66 & $0.92-3.00$ & 0.091 & & & \\
\hline \multicolumn{7}{|l|}{ CRP/Alb } \\
\hline$<0.05$ & 1 & & & & & \\
\hline$\geq 0.05$ & 1.91 & $1.40-2.60$ & $<0.001$ & & & \\
\hline \multicolumn{7}{|l|}{ CEA } \\
\hline$\leq 5 \mathrm{ng} / \mathrm{mL}$ & 1 & & & & & \\
\hline$>5 \mathrm{ng} / \mathrm{mL}$ & 1.76 & $1.16-2.68$ & 0.008 & & & \\
\hline
\end{tabular}

HR Hazard ratios, CI Confidence interval, BMI Body mass index, PNI Prognostic nutritional index, PI Prognostic index, GPS Glasgow Prognostic Score, mGPS Modified Glasgow Prognostic Score, NLR Neutrophil-lymphocyte ratio, PLR Platelet-lymphocyte ratio, CRP/Alb C-reactive protein/albumin, SII Systemic immune-inflammatory index 
Table 2 Multivariate analysis of prognostic factors in patients with advanced gastric cancer

\begin{tabular}{|c|c|c|c|c|c|c|}
\hline \multirow{2}{*}{$\begin{array}{l}\text { Clinicopathological } \\
\text { feature }\end{array}$} & \multicolumn{3}{|c|}{ Multivariate analysis DFS } & \multicolumn{3}{|c|}{ Multivariate analysis DSS } \\
\hline & $\overline{H R}$ & $95 \% \mathrm{Cl}$ & $p$ value & HR & $95 \% \mathrm{Cl}$ & $p$ value \\
\hline \multicolumn{7}{|l|}{ Age (years) } \\
\hline$\leq 60$ & 1 & & & 1 & & \\
\hline$>60$ & 1.61 & $1.10-2.35$ & 0.014 & 1.61 & $1.06-2.42$ & 0.024 \\
\hline \multicolumn{7}{|l|}{ PLR } \\
\hline$<154$ & 1 & & & 1 & & \\
\hline$\geq 154$ & 2.00 & $1.40-2.84$ & $<0.001$ & 2.09 & $1.43-3.04$ & $<0.001$ \\
\hline \multicolumn{7}{|l|}{ TNM } \\
\hline$\|$ & 1 & & & 1 & & \\
\hline III & 4.46 & $2.51-7.91$ & $<0.001$ & 5.94 & $3.01-11.74$ & $<0.001$ \\
\hline \multicolumn{7}{|l|}{ Chemotherapy } \\
\hline No & 1 & & & 1 & & \\
\hline Yes & 0.70 & $0.48-1.04$ & 0.074 & 0.66 & $0.45-0.99$ & 0.043 \\
\hline
\end{tabular}

DFS Disease-free survival, DSS Disease-specific survival, HR Hazard ratios, CI Confidence interval, $P L R$ Platelet-lymphocyte ratio
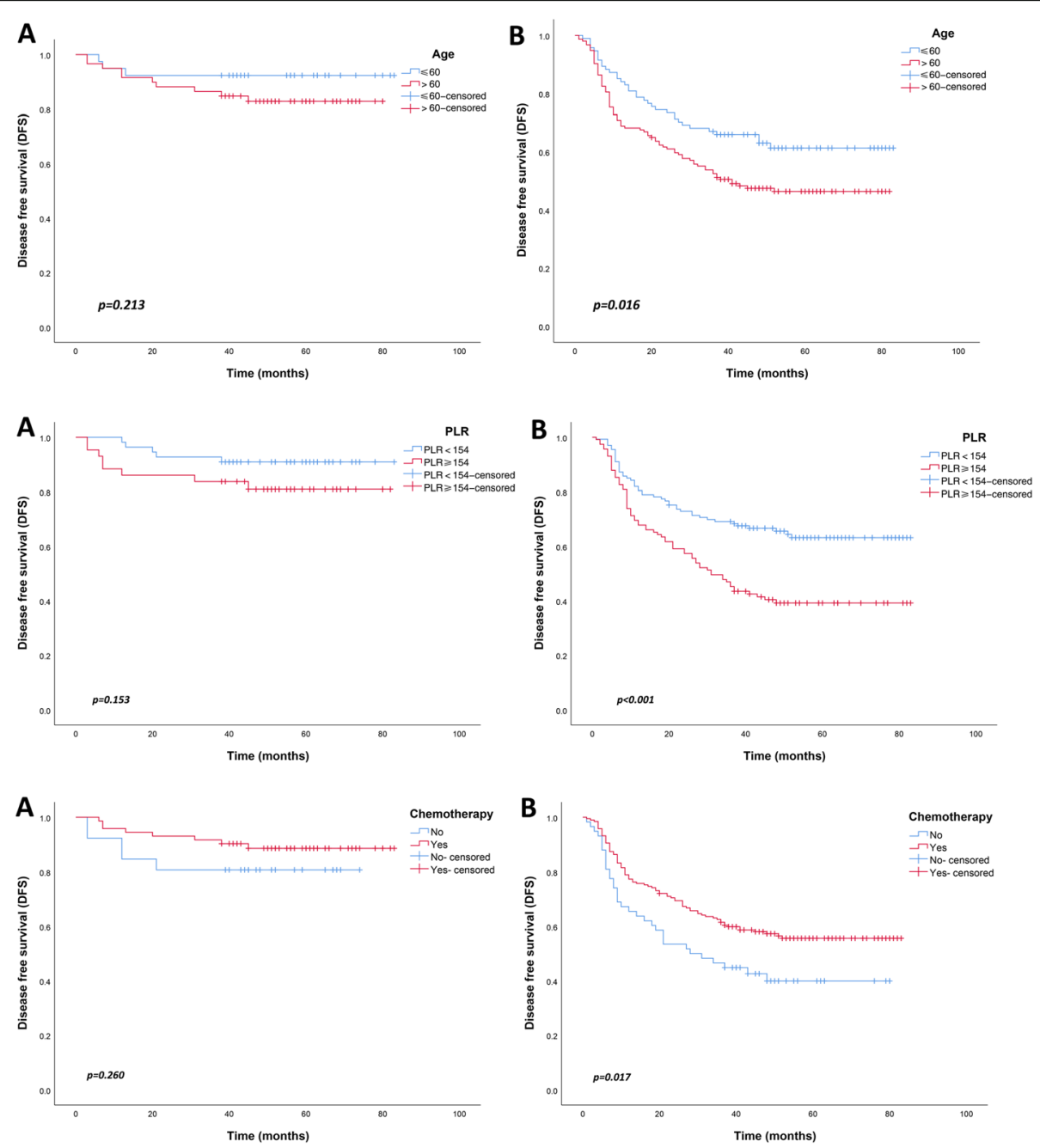

Fig. 1 Disease-free survival (DFS) of patients with advanced gastric cancer according to the risk factors. (a) stage II; (b) stage III 

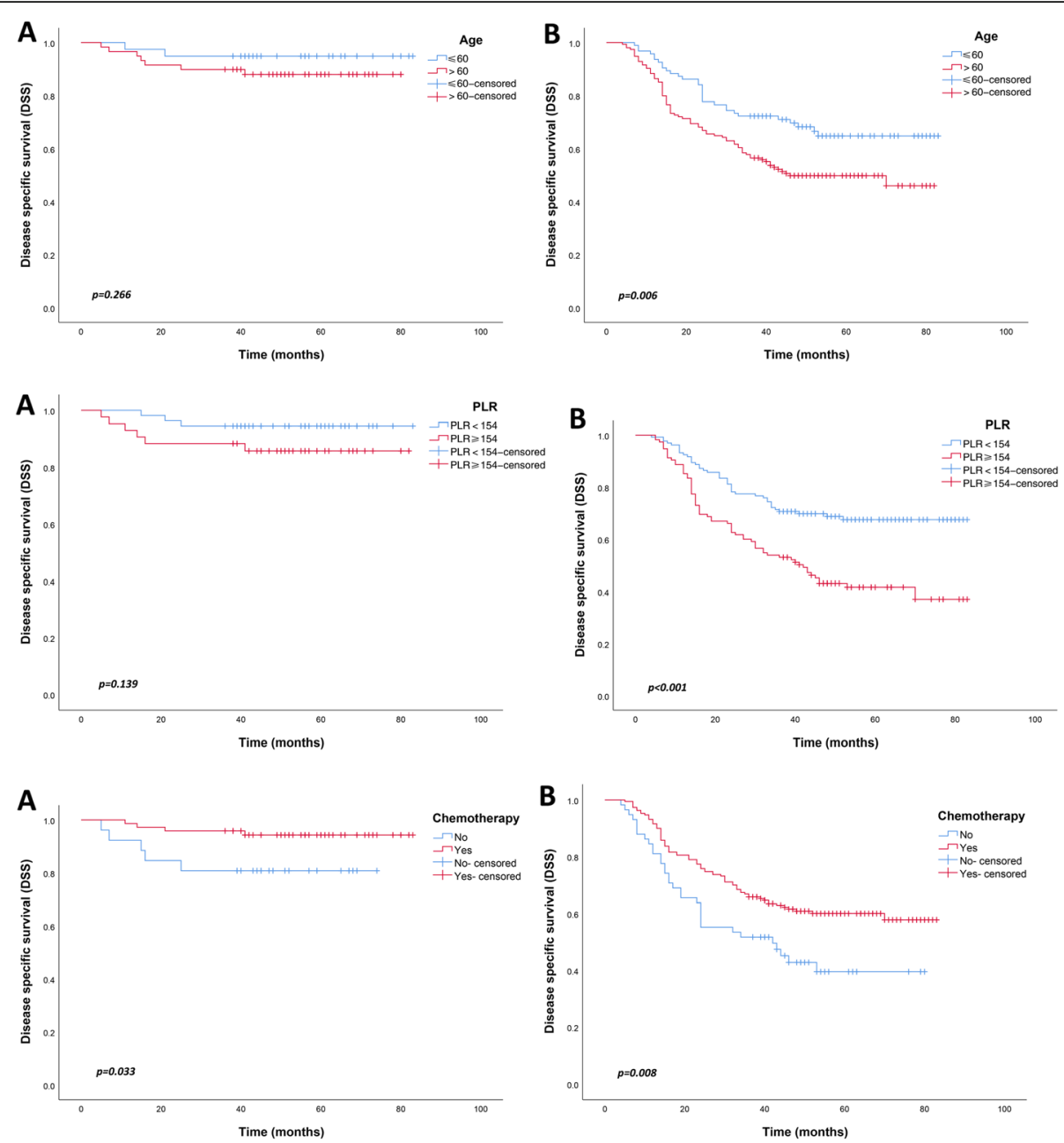

Fig. 2 Disease-specific survival (DSS) of patients with advanced gastric cancer according to the risk factors. (a) stage II; (b) stage III

inconsistent results, particularly on the significance of each inflammatory index and the threshold value.

Therefore, in addition to the peripheral blood-derived inflammation markers reported in previous studies, this study further explored the prognostic value of some conventional systemic inflammatory marker in patients with GC. We prospectively analyzed 598 GC patients after radical surgery and found that independent risk factors for poor prognosis of GC included age, $\mathrm{T}$ stage, $\mathrm{N}$ stage, number of lymph nodes retrieved, and PLR. Currently, TNM staging is the standard prognostic tool for GC. Given the excellent prognosis of early gastric cancer, our focus was to analyze the prognosis of patients with AGC (stage II/III). Many studies have demonstrated that the number of lymph node dissection has an impact on the prognosis of GC; therefore, we further excluded patients with an inadequate amount of lymph node dissection (less than [16, 24-26]).

Independent risk factors for AGC included age, PLR, TNM stages, and chemotherapy. Previous studies have investigated the effect of age on GC prognosis; however, most of these studies found no significant association between age and GC prognosis [27-29]. Takatsu analyzed $5000 \mathrm{GC}$ cases and found that early-onset GC (age $<40$ years) was likely to present lymph node metastases. But the survival rate of young GC patients was equivalent to that of older GC patients (age $\geq 60$ years) [30]. In the present study, older GC patients (age $>60$ years) had a worse prognosis, which was closely related to tumor recurrence.

Nutritional status is associated with survival in patients with malignant tumors, including GC. Preoperative underweight and low PNI are considered poor prognostic factors. Park suggested that careful nutritional intervention after surgery could improve the survival rate [9]. Besides, a meta-analysis concluded that a low PNI is significantly associated with poor overall survival except for stage IV GC [31]. However, consistent with the results of Li et al. [32], we observed that PNI was not associated with prognosis. Also, our results did not show a 
Table 3 Association between PLR and the patients' characteristics

\begin{tabular}{|c|c|c|c|}
\hline & PLR $<154$ & PLR $\geq 154$ & $p$ value \\
\hline Age (year) & & & 0.223 \\
\hline$\leq 60$ & 78 & 55 & \\
\hline$>60$ & 110 & 103 & \\
\hline Gender & & & 0.818 \\
\hline Male & 128 & 105 & \\
\hline Female & 60 & 53 & \\
\hline Tumor location & & & 0.941 \\
\hline Upper third & 26 & 47 & \\
\hline Middle third & 23 & 17 & \\
\hline Lower third & 133 & 116 & \\
\hline Two-thirds or more & 6 & 4 & \\
\hline Tumor size & & & $<0.001$ \\
\hline$\leq 5 \mathrm{~cm}$ & 135 & 73 & \\
\hline$>5 \mathrm{~cm}$ & 53 & 85 & \\
\hline Histologic type & & & 0.156 \\
\hline Differentiated & 74 & 75 & \\
\hline Undifferentiated & 114 & 83 & \\
\hline Perineural invasion & & & 0.194 \\
\hline Absence & 79 & 78 & \\
\hline Presence & 109 & 80 & \\
\hline Lymphovascular invasion & & & 0.509 \\
\hline Absence & 77 & 59 & \\
\hline Presence & 111 & 99 & \\
\hline T category & & & 0.835 \\
\hline $\mathrm{T} 1$ & 7 & 4 & \\
\hline $\mathrm{T} 2$ & 15 & 10 & \\
\hline T3 & 7 & 6 & \\
\hline T4a & 153 & 135 & \\
\hline $\mathrm{T} 4 \mathrm{~b}$ & 6 & 3 & \\
\hline N category & & & 0.416 \\
\hline NO & 35 & 28 & \\
\hline N1 & 39 & 29 & \\
\hline $\mathrm{N} 2$ & 58 & 41 & \\
\hline N3a & 45 & 43 & \\
\hline N3b & 11 & 17 & \\
\hline CEA & & & 0.275 \\
\hline$\leq 5 \mathrm{ng} / \mathrm{mL}$ & 166 & 133 & \\
\hline$>5 \mathrm{ng} / \mathrm{mL}$ & 22 & 25 & \\
\hline
\end{tabular}

correlation between fibrinogen and the prognosis of GC. Recent studies have shown that fibrinogen is one of the risk factors for poor prognosis in upper gastric cancer [33]. Fibrinogen is the primary acute-phase protein, and as a critical component of the hemostatic system, it regulates the systemic inflammatory state and cancer progression. However, its clinical significance in the prognosis of GC has not been elucidated.

The NLR and PLR are the most extensively studied markers of peripheral blood-derived inflammation, which are associated with the prognosis of GC. Accumulating evidence has shown that NLR and PLR are associated with distant metastases during GC progression [34, 35]. Kim reported that although both the PLR and NLR could reflect the prognosis, the NLR was more predictive of overall survival than the PLR in GC [36]. Also, they suggested that NLR and PLR might be associated with lymph node metastasis in early gastric cancer [37]. On the contrary, Zhu et al. indicated that NLR and PLR could not predict lymph node metastasis and prognosis in early gastric cancer [38]. In the present study, PLR was significantly correlated with the prognosis of GC, but there was no statistical difference between NLR and prognosis of GC. This observation seems to be inconsistent with previous studies, but the exact mechanism is still unclear. However, the inconsistencies could be because most previous studies focused on overall survival as the primary outcome, whereas herein, we used tumor recurrence and tumor-related mortality as observational indicators, which seem to be more accurate. In addition, the clinicopathological characteristics were similar between the PLR elevating group (PLR $<154$ ) and the PLR decreasing group (PLR $\geq 154$ ), except for tumor size, which further suggested that PLR might influence the prognosis of tumor through other mechanisms. A recent meta-analysis has revealed that PLR is associated with prognosis of GC [39].

Abnormal levels of CRP and Alb have been related to poor prognosis of tumor patients. It is noteworthy that the combinations of these two indicators can enhance the accuracy to predict the recurrence of multiple tumors. Among them, the most common evaluation criteria after combination include GPS and CRP/Alb. Besides, many studies have used GPS to predict the prognosis of various tumors, including GC. Hsueh recently recommended the use of GPS as a predictive and prognostic factor in patients with GC. A significant correlation was observed between the GPS, short-term postoperative complications, and long-term survival outcomes in patients with GC undergoing D2 gastrectomy [40]. Some studies have indicated that GPS and mGPS, used either alone or in combination, represent an independent prognostic factor for long-term outcome in resected GC [41, 42]. However, Walsh's results did not show a correlation between prognosis of patients and mGPS levels, although mGPS was associated with advanced GC stage [17]. Liu et al. retrospectively analyzed 455 patients with resectable GC and showed that CRP/ Alb, rather than GPS and mGPS, was associated with 

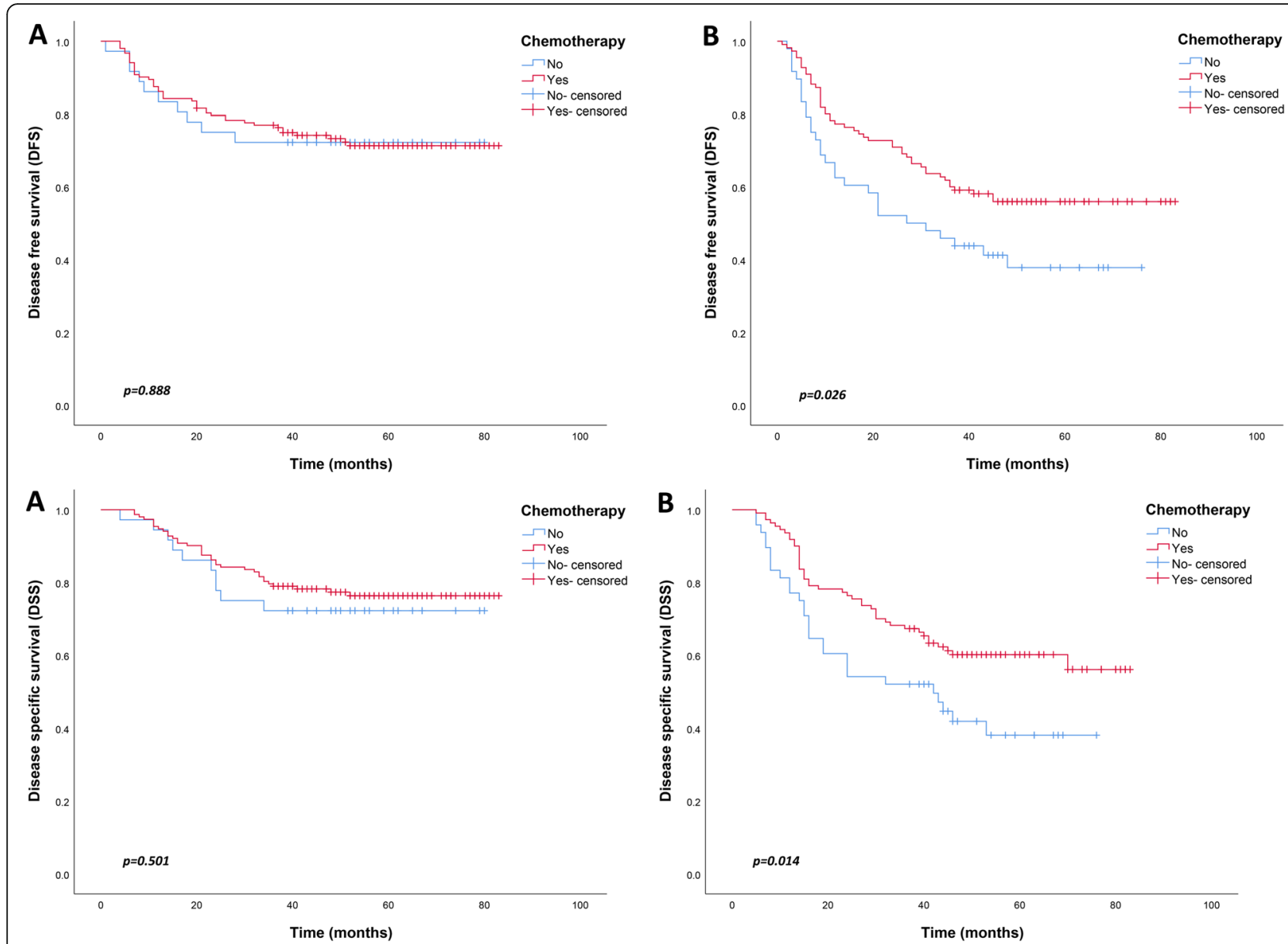

Fig. 3 Comparison of survival curves between patients with or without chemotherapy in advanced gastric cancer. (a) PLR $<154$; (b) PLR $\geq 154$

overall survival [43]. Similarly, Xu [12] and Lu [44] et al. also observed that $\mathrm{CRP} / \mathrm{Alb}$ and $\mathrm{CRP} /$ prealbumin were associated with recurrence of GC based on the data from a phase III randomized clinical trial. On the contrary, our results showed that although CRP/Alb and GPS were associated with prognosis in the univariate analysis, the multivariate analysis showed that both were not related to long-term survival.

Consistent with the results of previous clinical trials, our findings showed that chemotherapy could significantly improve the prognosis of AGC, especially for patients with stage III GC $[45,46]$. Moreover, many studies have investigated the correlation between peripheral blood-derived inflammation markers and the effects of chemotherapy, to guide the selection of chemotherapy-sensitive patients. A study suggested that the sensitivity of chemotherapy (oxaliplatin/5-fluorouracil combination) might be closely related to NLR, PLR, and their changes in metastatic gastric cancer [47]. Hirahara believed that the combination of NLR and PLR might be more effective in predicting the chemotherapy response in patients with metastatic gastric cancer [48]. Besides, Tang [49] and Chen [50] concluded that PLR could predict the efficacy of neoadjuvant chemotherapy of GC patients treated with oxaliplatin and capecitabine regimens. In the current study, PLR effectively predicted adjuvant chemotherapy (oxaliplatin/5-fluorouracil combination) response in patients with AGC after surgery. For patients with PLR $\geq 154$, chemotherapy significantly improved long-term survival, including DFS and DSS; however, patients with PLR $<154$ did not benefit from adjuvant chemotherapy. Thus, we recommend that AGC patients with PLR $\geq 154$ should actively receive adjuvant chemotherapy (oxaliplatin/5-fluorouracil combination) after surgery, whereas patients with PLR $<154$ need to be cautious when choosing adjuvant chemotherapy.

This study systematically evaluated the relationship between peripheral blood-derived inflammation markers and the prognosis of GC. Notably, the potential ability of inflammatory markers to predict the effects of chemotherapy was further demonstrated. However, this study had a few limitations. Importantly, this was an observational study and was therefore influenced by other confounding factors. For example, lymphocytes, neutrophils, CRP and others may be affected by complications such 
as chronic bronchitis, pneumonia. The time of collection of blood samples is not fixed, which may affect the test results. Also, the sample size was relatively small, and this may have reduced the reliability of the findings. Thus, these results need to be further validated by large multicenter randomized clinical trials.

\section{Conclusion}

Our findings show that PLR is significantly correlated with the prognosis of GC, especially for stage III. That is, higher PLR $(\geq 154)$ is an independent risk factor for poor long-term survival rate of GC patients. Moreover, PLR can be used to predict adjuvant chemotherapy (oxaliplatin/5-fluorouracil combination) response in patients with GC after surgery.

\section{Supplementary information}

Supplementary information accompanies this paper at https://doi.org/10. 1186/s12893-020-00884-8.

Additional file 1: Table S1. Baseline clinicopathological characteristics.

Additional file 2: Table S2. Optimal cutoff analysis.

\section{Abbreviation}

GC: Gastric cancer; AGC: Advanced gastric cancer; UICC: International Union Against Cancer; AJCC: American Joint Committee on Cancer;

NLR: Neutrophil-lymphocyte ratio; PLR: Platelet lymphocyte ratio; SII: Systemic immune-inflammation index; CRP/Alb: C-reactive protein/albumin ratio; GPS: Glasgow Prognostic Score; mGPS: Modified Glasgow Prognostic Score; BMI: Body mass index; PNI: Prognostic nutritional index; PI: Prognostic index; DFS: Disease-free survival; DSS: Disease-specific survival; ROC: Receiver operating characteristics; HR: Hazard ratios; Cl: Confidence intervals

\section{Acknowledgements}

Not applicable.

\section{Authors' contributions}

Conceptualization: DW; Data curation: LG, MW, and XC; Formal analysis: LG, $J M$; Investigation: KZ, DMN, and PC; Writing original draft: LY, FM, and LG. All authors have read and approved the manuscript.

\section{Funding}

This study was supported by Ningbo Clinical Research Center for Digestive System Tumors (Grant No. 2019A21003), Key Laboratory of Diagnosis and Treatment of Digestive System Tumors of Zhejiang Province (Grant No. 2019E10020), and Oncology Key Special Subject of Ningbo (2016-55). All the funders were authors of the study, and there was no conflict of interest.

\section{Availability of data and materials}

We state that all the raw data for this study have been provided.

\section{Ethics approval and consent to participate}

This study was approved by the Ethics Committee of the HwaMei Hospital and the University of Chinese Academy of Sciences (approval NO. PJ-NBEYKY-2019-153-01).

\section{Consent for publication}

Not applicable.

\section{Competing interests}

The authors declare no conflict of interest.

\section{Author details}

'Department of General Surgery, HwaMei Hospital, University of Chinese Academy of Sciences, Ningbo, Zhejiang, China. ${ }^{2}$ Key Laboratory of Diagnosis and Treatment of Digestive System Tumors of Zhejiang Province, Ningbo, Zhejiang, China. ${ }^{3}$ Ningbo Clinical Research Center for Digestive System Tumors, Ningbo, Zhejiang, China. ${ }^{4}$ Infection Department, Ningbo Yinzhou No. 2 Hospital, Ningbo, Zhejiang, China. ${ }^{5}$ Intensive Care Unit, Ningbo Yinzhou No. 2 Hospital, Ningbo, Zhejiang, China. ${ }^{6}$ The Second Clinical Medical College, Zhejiang Chinese Medical University, Hangzhou, Zhejiang, China. ${ }^{7}$ Medical College of Ningbo University, Ningbo, Zhejiang, China. ${ }^{8}$ Department of Clinical Laboratory, HwaMei Hospital, University of Chinese Academy of Sciences, Northwest Street 41, Haishu District, Ningbo 315010, Zhejiang, China.

Received: 4 July 2020 Accepted: 24 September 2020

Published online: 02 October 2020

\section{References}

1. Allemani C, Matsuda T, Di Carlo V, et al. Global surveillance of trends in cancer survival 2000-14 (CONCORD-3): analysis of individual records for 37 513025 patients diagnosed with one of 18 cancers from 322 populationbased registries in 71 countries. Lancet. 2018:391:1023-75.

2. Macdonald JS, Smalley SR, Benedetti J, et al. Chemoradiotherapy after surgery compared with surgery alone for adenocarcinoma of the stomach or gastroesophageal junction. N Engl J Med. 2001;345:725-30.

3. Japanese Gastric Cancer Association. Japanese gastric cancer treatment guidelines 2014 (ver. 4). Gastric Cancer. 2017;20:1-19.

4. Shi H, Jiang Y, Cao H, et al. Nomogram based on systemic immuneinflammation index to predict overall survival in gastric Cancer patients. Dis Markers. 2018:2018:1787424.

5. Miyamoto R, Inagawa S, Sano N, et al. The neutrophil-to-lymphocyte ratio (NLR) predicts short-term and long-term outcomes in gastric cancer patients. Eur J Surg Oncol. 2018:44:607-12.

6. Zhang LX, Wei ZJ, Xu AM, Zang JH. Can the neutrophil-lymphocyte ratio and platelet-lymphocyte ratio be beneficial in predicting lymph node metastasis and promising prognostic markers of gastric cancer patients? Tumor maker retrospective study. Int J Surg. 2018;56:320-7.

7. Petrillo A, Laterza MM, Tirino G, et al. Systemic-inflammation-based score can predict prognosis in metastatic gastric cancer patients before first-line chemotherapy. Future Oncol. 2018;14:2493-505.

8. Zheng L, Zou K, Yang C, et al. Inflammation-based indexes and clinicopathologic features are strong predictive values of preoperative circulating tumor cell detection in gastric cancer patients. Clin Transl Oncol. 2017:19:1125-32.

9. Park SH, Lee $\mathrm{S}$, Song JH, et al. Prognostic significance of body mass index and prognostic nutritional index in stage II/III gastric cancer. Eur J Surg Oncol. 2020;46:620-5.

10. Jagadesham VP, Lagarde SM, Immanuel A, Griffin SM. Systemic inflammatory markers and outcome in patients with locally advanced adenocarcinoma of the oesophagus and gastro-oesophageal junction. $\mathrm{Br}$ J Surg. 2017;104:401-7.

11. Huang $Z$, Liu $Y$, Yang $C$, et al. Combined neutrophil/platelet/lymphocyte/ differentiation score predicts chemosensitivity in advanced gastric cancer. BMC Cancer. 2018;18:515.

12. $\mathrm{Xu} B B, L \mathrm{~L} J$, Zheng $\mathrm{ZF}$, et al. The predictive value of the preoperative Creactive protein-albumin ratio for early recurrence and chemotherapy benefit in patients with gastric cancer after radical gastrectomy: using randomized phase III trial data. Gastric Cancer. 2019;22:1016-28.

13. Bang YJ, Kim YW, Yang HK, et al. Adjuvant capecitabine and oxaliplatin for gastric cancer after D2 gastrectomy (CLASSIC): a phase 3 open-label, randomised controlled trial. Lancet. 2012;379:315-21.

14. Boku N, Ryu MH, Kato K, et al. Safety and efficacy of nivolumab in combination with S-1/capecitabine plus oxaliplatin in patients with previously untreated, unresectable, advanced, or recurrent gastric/ gastroesophageal junction cancer: interim results of a randomized, phase II trial (ATTRACTION-4). Ann Oncol. 2019;30:250-8.

15. Ryu MH, Yoo C, Kim JG, et al. Multicenter phase II study of trastuzumab in combination with capecitabine and oxaliplatin for advanced gastric cancer. Eur J Cancer. 2015;51:482-8.

16. Rao S, Starling N, Cunningham D, et al. Matuzumab plus epirubicin, cisplatin and capecitabine (ECX) compared with epirubicin, cisplatin and 
capecitabine alone as first-line treatment in patients with advanced oesophago-gastric cancer: a randomised, multicentre open-label phase II study. Ann Oncol. 2010;21:2213-9.

17. Walsh SM, Casey S, Kennedy R, et al. Does the modified Glasgow prognostic score (mGPS) have a prognostic role in esophageal cancer? J Surg Oncol. 2016;113:732-7.

18. Song S, Li C, Li S, et al. Derived neutrophil to lymphocyte ratio and monocyte to lymphocyte ratio may be better biomarkers for predicting overall survival of patients with advanced gastric cancer. Onco Targets Ther. 2017; 10:3145-54

19. Liu Y, Chen S, Zheng C, et al. The prognostic value of the preoperative Creactive protein/albumin ratio in ovarian cancer. BMC Cancer. 2017;17:285.

20. Cannon NA, Meyer J, lyengar $P$, et al. Neutrophil-lymphocyte and plateletlymphocyte ratios as prognostic factors after stereotactic radiation therapy for early-stage non-small-cell lung cancer. J Thorac Oncol. 2015;10:280-5.

21. Yu L, Lv CY, Yuan AH, et al. Significance of the preoperative neutrophil-tolymphocyte ratio in the prognosis of patients with gastric cancer. World J Gastroenterol. 2015;21:6280-6.

22. Hu D, Zhang H, Lin X, et al. Elevated preoperative neutrophil-to-lymphocyte ratio can predict poor survival in early stage gastric cancer patients receiving radical gastrectomy: the Fujian prospective investigation of cancer (FIESTA) study. J Cancer. 2017:8:1214-22.

23. Liu $X, W u Z$, Lin $E$, et al. Systemic prognostic score and nomogram based on inflammatory, nutritional and tumor markers predict cancer-specific survival in stage II-III gastric cancer patients with adjuvant chemotherapy. Clin Nutr. 2019;38:1853-60

24. Kulig J, Sierzega M, Kolodziejczyk P, Popiela T. Ratio of metastatic to resected lymph nodes for prediction of survival in patients with inadequately staged gastric cancer. Br J Surg. 2009;96:910-8.

25. Lee HJ, Hyung WJ, Yang HK, et al. Short-term outcomes of a multicenter randomized controlled trial comparing laparoscopic distal Gastrectomy with D2 lymphadenectomy to open distal Gastrectomy for locally advanced gastric Cancer (KLASS-02-RCT). Ann Surg. 2019;270:983.

26. Shoda K, Ichikawa D, Arita T, et al. Risk stratification according to the Total number of factors that meet the indication criteria for radical lymph node dissection in patients with early gastric Cancer at risk for lymph node metastasis. Ann Surg Oncol. 2016;23:792-7.

27. Lu J, Chen Y, Liu Y, et al. Clinical significance of prognostic score based on age, tumor size, and grade in gastric cancer after gastrectomy. Cancer Manag Res. 2018;10:4279-86.

28. Song M, Kang D, Yang JJ, et al. Age and sex interactions in gastric cancer incidence and mortality trends in Korea. Gastric Cancer. 2015;18:580-9.

29. Zhang J, Gan L, Xu MD, et al. The prognostic value of age in non-metastatic gastric cancer after gastrectomy: a retrospective study in the U.S. and China. J Cancer. 2018;9:1188-99.

30. Takatsu Y, Hiki N, Nunobe S, et al. Clinicopathological features of gastric cancer in young patients. Gastric Cancer. 2016;19:472-8.

31. Yang $Y$, Gao $P$, Song $Y$, et al. The prognostic nutritional index is a predictive indicator of prognosis and postoperative complications in gastric cancer: a meta-analysis. Eur J Surg Oncol. 2016;42:1176-82.

32. Li J, Xu R, Hu DM, et al. Prognostic nutritional index predicts outcomes of patients after Gastrectomy for Cancer: a systematic review and metaanalysis of nonrandomized studies. Nutr Cancer. 2019:71:557-68.

33. Cong $X$, Li S, Zhang $Y$, et al. The combination of preoperative fibrinogen and neutrophil-lymphocyte ratio is a predictive prognostic factor in esophagogastric junction and upper gastric cancer. J Cancer. 2019;10:551826.

34. Miyatani $\mathrm{K}$, Saito $\mathrm{H}$, Kono $\mathrm{Y}$, et al. Combined analysis of the pre- and postoperative neutrophil-lymphocyte ratio predicts the outcomes of patients with gastric cancer. Surg Today. 2018;48:300-7.

35. Nora I, Shridhar R, Huston J, Meredith K. The accuracy of neutrophil to lymphocyte ratio and platelet to lymphocyte ratio as a marker for gastrointestinal malignancies. J Gastrointest Oncol. 2018;9:972-8.

36. Kim EY, Lee JW, Yoo HM, et al. The platelet-to-lymphocyte ratio versus neutrophil-to-lymphocyte ratio: which is better as a prognostic factor in gastric Cancer? Ann Surg Oncol. 2015;22:4363-70.

37. Lou N, Zhang $L$, Chen XD, et al. A novel scoring system associating with preoperative platelet/lymphocyte and clinicopathologic features to predic lymph node metastasis in early gastric cancer. J Surg Res. 2017;209:153-61.

38. Zhu GS, Tian SB, Wang $H$, et al. Preoperative neutrophil lymphocyte ratio and platelet lymphocyte ratio cannot predict lymph node metastasis and prognosis in patients with early gastric Cancer: a single institution investigation in China. Curr Med Sci. 2018;38:78-84.

39. Cao W, Yao X, Cen D, et al. The prognostic role of platelet-to-lymphocyte ratio on overall survival in gastric cancer: a systematic review and metaanalysis. BMC Gastroenterol. 2020;20:16.

40. Hsueh SW, Liu KH, Hung CY, et al. Significance of the Glasgow prognostic score in predicting the postoperative outcome of patients with stage III gastric Cancer. J Clin Med. 2019;8:1448.

41. Melling N, Gruning A, Tachezy M, et al. Glasgow prognostic score may be a prognostic index for overall and perioperative survival in gastric cancer without perioperative treatment. Surgery. 2016;159:1548-56.

42. Jiang $X$, Hiki N, Nunobe $S$, et al. Prognostic importance of the inflammationbased Glasgow prognostic score in patients with gastric cancer. Br J Cancer. 2012;107:275-9.

43. Liu X, Sun X, Liu J, et al. Preoperative C-reactive protein/albumin ratio predicts prognosis of patients after curative resection for gastric Cancer. Transl Oncol. 2015;8:339-45.

44. Lu J, Xu BB, Zheng ZF, et al. CRP/prealbumin, a novel inflammatory index for predicting recurrence after radical resection in gastric cancer patients: post hoc analysis of a randomized phase III trial. Gastric Cancer. 2019;22: 536-45.

45. Koizumi W, Takiuchi H, Yamada Y, et al. Phase II study of oxaliplatin plus S-1 as first-line treatment for advanced gastric cancer (G-SOX study). Ann Oncol. 2010;21:1001-5.

46. Al-Batran SE, Hartmann JT, Hofheinz R, et al. Biweekly fluorouracil, leucovorin, oxaliplatin, and docetaxel (FLOT) for patients with metastatic adenocarcinoma of the stomach or esophagogastric junction: a phase II trial of the Arbeitsgemeinschaft Internistische Onkologie. Ann Oncol. 2008;19:1882-7.

47. Lee S, Oh SY, Kim SH, et al. Prognostic significance of neutrophil lymphocyte ratio and platelet lymphocyte ratio in advanced gastric cancer patients treated with FOLFOX chemotherapy. BMC Cancer. 2013;13:350.

48. Hirahara T, Arigami T. Combined neutrophil-lymphocyte ratio and plateletlymphocyte ratio predicts chemotherapy response and prognosis in patients with advanced gastric cancer. BMC Cancer. 2019;19:672.

49. Tang C, Cheng X, Yu S, et al. Platelet-to-lymphocyte ratio and lymphocyte-towhite blood cell ratio predict the efficacy of neoadjuvant chemotherapy and the prognosis of locally advanced gastric cancer patients treated with the oxaliplatin and capecitabine regimen. Onco Targets Ther. 2018;11:7061-75.

50. Chen L, Hao Y, Cong $X$, et al. Peripheral venous blood platelet-tolymphocyte ratio (PLR) for predicting the survival of patients with gastric Cancer treated with SOX or XELOX regimen Neoadjuvant chemotherapy. Technol Cancer Res Treat. 2019:18:1533033819829485.

\section{Publisher's Note}

Springer Nature remains neutral with regard to jurisdictional claims in published maps and institutional affiliations.
Ready to submit your research? Choose BMC and benefit from:

- fast, convenient online submission

- thorough peer review by experienced researchers in your field

- rapid publication on acceptance

- support for research data, including large and complex data types

- gold Open Access which fosters wider collaboration and increased citations

- maximum visibility for your research: over $100 \mathrm{M}$ website views per year

At $\mathrm{BMC}$, research is always in progress.

Learn more biomedcentral.com/submissions 